\title{
Gombrowicz tra esilio e straduzione Vera Horn
}

ABSTRACT: L'esilio argentino dello scrittore polacco Witold Gombrowiz raccontato da Laura Pariani nel romanzo La straduzione; il tentativo di tradursi; il tentativo di tradurre il Ferdyduke in spagnolo e conquistare pubblico; critica e traduzione nel mondo argentino; la straduzione come fallimento. PAROLE CHIAVE: Laura Pariani; Witold Gombrowicz; La straduzione; Argentina; esilio; traduzione; scrittore migrante.

Sei un emigrante, non te li puoi permettere gli ideali.

Sto cercando di vivere come se fossi una persona libera. Almeno quella dignità mi sarà concessa.

Gli aggettivi non varcano $i$ continenti.

Eva Hoffman, Lost in translation. $A$ life in a new language

A tre anni dal suo ritorno in Europa e poco prima della morte, Witold Gombrowicz scriveva nel suo diario: "Mi risulta doloroso che rimanga così poco del mio soggiorno argentino. Dove sono quelli che possono narrare la mia storia, descrivere e raccontare come io ero?"' Ci ha provato Laura Pariani, in La straduzione, un romanzo che racconta borgesianamente il soggiorno argentino di Gombrowicz,

1. Witold Gombrowicz, Diario, apud Laura Pariani, La straduzione, Milano, Rizzoli, 2004 (risvolto di copertina). 
anche se in realtà i due scrittori erano divisi da concezioni letterarie molto diverse. Gombrowicz spiegava così la sua distanza da Borges: "Borges e io siamo agli antipodi. Lui è radicato nella cultura, io nella vita". "Niente ha significato se non viene raccontato"3, sembra dire l'autrice in risposta alle ansie di Gombrowicz. Questo procedimento non è nuovo nell'opera della Pariani, che in La foto di Orta aveva già cercato di ricostruire romanzescamente il soggiorno di Nietzsche e Lou Salomé sul lago d'Orta.

Nel romanzo di Pariani, Gombrowicz, allora giovane scrittore polacco, sbarca in Argentina per un breve soggiorno, ma al momento del ritorno decide di restarci. In Europa c'era la guerra e la sua Polonia era stata invasa da Hitler. Senza un lavoro, senza soldi in tasca, senza un obiettivo ben preciso, lo scrittore decide di fare dell'Argentina la sua patria in quel momento, con grande libertà. All'inizio Gombrowicz vive una vita da esule volontario, ma costretto ai più svariati espedienti pur di avere un piatto di cibo e un posto dove dormire. Non gli manca solo la struttura di base, ma anche il senso del gruppo e dell'appartenenza: Gombrowicz vive infatti in un quasi totale isolamento, con poche ed eccentriche frequentazioni, e comunque appartato dai circoli letterari. A poco a poco si riavvicina alla letteratura, ma subisce subito innumerevoli delusioni, dall'articolo firmato da un altro ai libri e articoli respinti, o anche alla frequentazione di "salotti-tutta-cultura" più o meno formali, sia che si svolgessero in un bar, sia in una casa lussuosamente arredata con tanti poster di prime teatrali e le ultime novità francesi (un riferimento al salotto Ocampo-Borges). Alla fine una donna influente ottiene dall'Editorial Argos la possibilità di tradurre in spagnolo il suo Ferdydurke, scritto e pubblicato in Polonia. A questo punto, comincia "il sogno di una pazza straduzione", come la chiama l'autrice verso la fine del romanzo.

All'inizio la traduzione viene affidata a Gombrowicz, che però non riesce nell'impresa, tra insonnie e affanni. Il suo "stentato castellano" si arenava facilmente nelle insidie della traduzione e ogni difficoltà gli sembrava "amaramente insolubile", tale

2. Witold Gombrowicz, Testamento, trad. Vera Verdiani, Milano, Feltrinelli, 2004, p. 99.

3. Laura Pariani, La straduzione, op. cit., p. 46. 
da fargli considerare la faccenda come un ulteriore "fallimento personale". Siccome Gombrowicz decide di rifiutare la proposta per la sua scarsa dimestichezza con lo spagnolo, lo scrittore cubano Virgilio Piñera propone di formare un comité di traduzione che lo sostenga nell'avventura della traduzione: Gombrowicz racconterebbe a Piñera e agli altri collaboratori più o meno fissi il testo e questi cercherebbero di tradurlo in uno spagnolo accettabile, passando magari per il francese, nel caso lo spagnolo imperfetto di Gombrowicz non riuscisse a far capire qualche brano o concetto: "Sarà un'esperienza mai tentata in nessun'altra parte del mondo: tradurre da una lingua che nessuno dei traduttori né conosce né parla. [...] Pensate: cubano, francese, polacco e argentino, una mescolanza esplosiva"4 E cosi fu. Le riunioni, ben descritte da Pariani, che si rifa all'universo variopinto che contrassegna le frequentazioni di Gombrowicz durante tutto il soggiorno argentino del romanzo, dagli intellettuali al "campionario umano di odori sulfuri di tabacco nero e piedi sudati", con enfasi negli ultimi, si svolgevano nel sotterraneo di un bar, il Rex, con l'ausilio dei compagni fissi, "tutti expatriados, extrangeros: detriti che le guerre o le dittature avevano depositato sulle rive del Río de la Plata", e di quelli sporadici, tra battute da biliardo scandite dall'alcool, discussioni filologiche e trovate esilaranti, come quando per tradurre la risata di una signora grassa, decidono per "scoppiò in un riso di cameriera pizzicata sul didietro", il tutto con metodo "assolutamente scientifico", come sottolinea Piñera, ma dove il senso può comunque essere un non-senso. A volte ci mettevano due ore per "definire le qualità dello sguardo di un commesso viaggiatore; a volte tutto si risolveva "con un'intuizione improvvisa".

La vicenda di Gombrowicz di esule volontario ai margini della società, il sogno della pazza straduzione toccano punti fondamentali del meccanismo della tradu-
4. Ib., p. 168.
5. Ib., p. 112
6. Ib., p. 164 .
7. I6., p. 181.
8. I6., p. 181. 
zione, come la questione della lingua, della traducibilità, la nozione di fedeltà, di letteralità, di invisibilità del traduttore e, soprattutto, della traduzione vissuta da uno scrittore emigrato, come il problema della (doppia) lingua, la difficile negoziazione con un altro sistema linguistico, la capacità di trapiantare un testo come un paese. Per il Gombrowicz parianesco si tratta, infatti, di un doppio trapianto, quello geografico e quello linguistico. C'è sempre, nella traduzione come nella migrazione, un attraversamento e una doppia appartenenza, un momento in cui la parola, così come il migrante, sono sospesi, non ancora fissati in un significante/significato più o meno stabile, in una specie di deriva. Gombrowicz, come scrittore migrante, vive la sua esperienza come traduzione, "passaggio fisico e letterario tra due realtà"9.

Prima ancora della traduzione, la cui avventura inizierà soltanto verso il tredicesimo capitolo, per Gombrowicz si presenta la condizione dell'esule, dell'emigrato, seppur volontario, ma non privo di ferite. La condizione dell'emigrante non prevede nessun riconoscimento: "Scrittore polacco, ha detto? Qui in Argentina è come essere italiano: qualcosa che non ha nessun prestigio e puzza di miseria immigratoria"10. Gombrowicz si sente imperfetto, incapace di imporsi al pubblico, diviso tra le cambiali e i rifiuti di pubblicazione ("nessuno mi prende sul serio"11). Finita la guerra, la soluzione più facile sarebbe tornare a casa, riprendere il filo lasciato qualche anno addietro. Questa situazione gli crea non poche perplessità: "Che bello se avesse sentito rinascere dentro di sé la parolina 'patria' e se fosse riuscito semplicemente a salire su una nave. Perché non se ne andava?" 12 . Alla fine lo scrittore conclude che "se scegliere l'Argentina nel '39 forse è stata una fuga, adesso so che ogni evasione è finita, il Sudamerica è diventato la mia 'realtà', e l'Europa per me è un nome che non designa più nessuno, triste parola che, se qualche volta si insinua ancora nella mia mente, è solo per insegnarmi che l'unica verità è la separazione... La Polonia

9. Vedi Silvia Albertazzi, Lo sguardo dell'altro. Le letterature postcoloniali, Roma, Carocci, 2000, p. 146.

10. Laura Pariani, La straduzione, op. cit., p. 51.

11. 16., p. 54.

12. Ib., p. 158. 
era ormai tanto lontana quanto i suoi sogni di ragazzo"13. Questa constatazione permette allo scrittore di "guardare il mondo con occhi nuovi"14 e di decidere di restare ancora in Argentina, paese che lascerà soltanto nel 1963. Gombrowicz era troppo scosso dagli avvenimenti storici; non era quindi possibile ritrasferirsi in Polonia. La guerra era passata, ma non la sua distruzione, e l'Argentina restava comunque una meta liberatoria: "Aveva bisogno di cielo, di arcobaleni, di orizzonti vasti"15. Da benestante e ben radicato nella lingua in Polonia, Gombrowicz passa a vivere la condizione dello straniero escluso, uomo senza patria in fuga dalla storia, senza una conoscenza linguistica idonea a portarlo in un livello di dignità: "solo e fracasado in mezzo a un'enorme città straniera" ${ }^{16}$.

Gombrowicz, nel vivere l'esilio, lo fa con leggerezza e con senso di libertà, ma allo stesso tempo con la pesantezza di una sensazione di sconfitta: " $\mathrm{E}$ questo il caso di uno scrittore in cui nessuno crede che un certo giorno, stanco di se stesso, ha deciso di seguire la propria vocazione di perdente assumendo il ruolo dell'esule a cui è espressamente proibito avere aspettative" 17 ; e con la consapevolezza dell'isolamento: "Da anni viveva appartato, solo; da desterrado, da straniero" ${ }^{18}$. I salotti letterari per bene non lo comprendono, lo marchiano come eccentrico e non lo accettano per quello che è: "I maneggioni della letteratura non sanno che farsene di chi si stacca dai loro soliti modi, il mio lavoro per loro non ha alcun valore, le mie incursioni dietro le linee per catturare la vita non hanno sortito nessun effetto, nessuno se n'è accorto [...]"19, anche per quanto riguarda il significato della letteratura: "Vi siete scordati della carne e del sangue che pulsa, anche i vostri libri puzzano di morto"20.

13. Ib., p. 160.

14. $16 .$, p. 160.

15. Ib., p. 157.

16. Ib., p. 158.

17. Ib., p. 157.

18. Ib., p. 130.

19. Ib., p. 149. 
Nello spiegare a un frequentatore del Rex che cos'è la traduzione, Gombrowicz la definisce nei seguenti termini: "Significa scegliere altre parole per dire le stesse cose" Ma sceglierle come?, obbietta l'interlocutore. "Trovare parole speciali: aspre, di quelle che scendano ruvide in gola; oppure dolci, come frutti canditi. A seconda della necessità" ${ }^{21}$, risponde lo scrittore. Le parole della traduzione sono scelte secondo criteri dinamici che si presentano ogniqualvolta si ponga l'occasione che ne determini la scelta. Lo scegliere si impone quale azione fondamentale del processo, giacché la traduzione non è mai un'operazione neutra. Piñera aveva anticipato che il comité avrebbe lavorato con criteri assolutamente scientifici: "Ogni parola sarà discussa sotto turti i suoi aspetti: il senso, il doppio senso, l'eufonia, la cadenza..."22, così come il giocatore esperto di biliardo "cercava l'angolo preciso per il colpo, la simmetria perfetta" ${ }^{\text {"23 }}$ Classico esempio di questa prerogativa è la discussione intorno alla traduzione della parola "cavallo" Un frequentatore del bar e, quindi, uno dei traduttori "sporadici", protesta che la parola potrebbe avere diverse accezioni, a seconda del sesso, dell'età, del colore, della capacità lavorativa, della resistenza della bestia, per non dimenticare le sfumature affettive. Il comité, al di là delle sedure strampalate, si fa forte dell'interpretazione come spunto per la traduzione, visto che parte da una conoscenza linguistica imperfetta, senza tralasciare però il perno intorno al quale ruotano molte delle discussioni, e cioè la parola, che dovrà significare il concetto espresso nella lingua d'arrivo, alla quale è dato un peso maggiore, essendo la lingua di partenza sconosciuta e tradotta. L'accostamento della traduzione all'interpretazione non è nuovo negli studi sull'argomento; Heidegger, Steiner e Gadamer, per citare alcuni esempi, avevano già sostenuto che la traduzione è un'interpretazione. Nel caso del Ferdydurke abbiamo una specie di traduzione di secondo grado: nel raccontare oralmente il resto polacco al comité, Gombrowicz compie un'operazione

20. Ib., p. 124.

21. Ib., Pp. 180-181.

22. Ib., p. 168.

23. Ib., p. 182. 
traduttoria; la sua non è che una traduzione, che verrà poi interpretata e ritradotta in uno "spagnolo letterario", come lo chiama Piñera. La traduzione portata avanti dal comité non privilegia il riscatto del significato originale, ma compie senz'altro un atto di produzione di significati.

Il lavoro del comité procedette pressappoco con questo ritmo fino alla conclusione del lavoro e finalmente la traduzione venne pubblicata, ma senza un grande risultato. Per Gombrowicz, questa pubblicazione significava la possibilità di essere finalmente compreso e accettato come scrittore, come si apprende, tra l'altro, dalle battute sul "romanzo argentino-romanzo polacco" e dall'aspettativa creatasi intorno all'imminente pubblicazione: "Penso alle persone che avrebbero letto le sue pagine e se ne sarebbero meravigliate, vide il volume sopra una bancarella, con le pagine lacere, impronte di dita [...]; un uomo con un grosso soprabito che si metteva il libro in tasca prima di fare un viaggio, un altro che gli scriveva una lettera"24. Aspettativa che viene subito e inevitabilmente delusa, come registra Pariani: "Col batticuore scrutava il viso dell'uomo, attento al voltare delle pagine, tremò al vedere come il 'suo lettore' sbuffando richiudeva il libro e lo buttava nella sua valigetta"25. L'insuccesso dell'impresa lo colloca nuovamente di fronte a questa condizione. La traduzione fallisce come strategia di inserimento nella cultura argentina e non riesce ad aprire le porte a "uomini e donne come cigni che, appena fiutavano nell'altro il diverso, l'anatrino, gli si gettavano contro, beccandolo a morte"26.

Nel romanzo della Pariani la traduzione diventa un modo di sfidare il concetto e la pratica stessa della traduzione, lasciando che il problema affiori nel movimento di passaggio da una versione all'altra. Ma non solo: la traduzione è anche una forma di leggere la migrazione, l'esilio, l'isolamento, l'incomunicabilità, la lingua della migrazione. Per Gombrowicz, straduzione e "desencuentro" si ritrovano sulla stessa via. Il capitolo "Desencuentro" narra una conversazione tra Gombrowicz e Roberto 
Arlt, in cui lo scrittore argentino esprime, attraverso le parole di un tango ("le parole sono come navi che solcano il mare; vanno da un'anima all'altra col rischio di naufragare" ${ }^{27}$ ), l'idea che la vita sia sostanzialmente "desencuentro", ciò̀, incapacità di capirsi veramente. Se la straduzione significa per Gombrowicz il fallimento di un sogno di affermazione e di riconoscimento, nonché il riscatto dalla miseria e dalla guerra, il "desencuentro" significa la solitudine dell'esilio, dell'essere appartato, del vivere da outsider. Il ritratto di Gombrowicz ad opera della Pariani mette in risalto il nesso esilio-straduzione; straduzione-desecuentro; emigrazione-fallimento; dispatrio-disperanza. Per l'autrice, l'unica verità che rimane per sempre è la sconfitta. Ma anche per il Gombrowicz degradato e "fracasado" della Pariani la scrittura può tuttavia fungere da mezzo di redenzione. Questo pensiero non è nuovo nell'opera della scrittrice, che già nel libro precedente (L'uovo di Gertrudina, 2003), affermava: "La letteratura può anche essere gesto di libertà, di salvezza, perfino di redenzione"28 In questo senso, non solo il soggiorno argentino gombrowcziano acquisisce il senso della memoria nella voce della scrittrice, ma lo scrittore stesso sembra trovare nella scrittura un senso alla sua esistenza senza "consuelo": "Io non chiedo a nessuno di restare con me. Né prometto pomi d'oro a chi mi leggerà. Il mio compito è sedere al tavolo in questa stanza a pensione e battere sui tasti della mia macchina da scrivere, per mettere dentro la pagina quanta più eternità possibile"29.

RESUMO: O exilio argentino do escritor polonês Witold Gombrowicz narrado por Laura Pariani no romance La straduzione; a tentativa de traduzir-se; a tentativa de traduzir o Ferdyduke em espanbol para conquistar público; crítica e tradufāo no mundo argentino; a "straduzione" como fracasso.

PALAVRAS-CHAVE: Laura Pariani; Witold Gombrowicz; La straduzione; Argentina; exilio; traduçäo; escritor migrante.

27. Ib., p. 58.

28. Laura Pariani, Luovo di Gertrudina, Milano, Rizzoli, 2003, p. 220.

29. Ib., pp. 201-202. 